\title{
PewLSTM: Periodic LSTM with Weather-Aware Gating Mechanism for Parking Behavior Prediction
}

\author{
Feng Zhang ${ }^{1}$, Ningxuan Feng ${ }^{2}$, Yani Liu ${ }^{1}$, Cheng Yang $^{3}$, Jidong Zhai ${ }^{2}$, \\ Shuhao Zhang ${ }^{4,5}$, Bingsheng $\mathbf{H e}^{5}$, Jiazao Lin $^{6}$ and Xiaoyong $\mathbf{D u}^{1}$ \\ ${ }^{1}$ Key Laboratory of Data Engineering and Knowledge Engineering (MOE), and School of Information, \\ Renmin University of China \\ ${ }^{2}$ Department of Computer Science and Technology, Tsinghua University, BNRist \\ ${ }^{3}$ School of Computer Science, Beijing University of Posts and Telecommunications \\ ${ }^{4}$ DIMA, Technische Universität Berlin \\ ${ }^{5}$ School of Computing, National University of Singapore \\ ${ }^{6}$ Department of Information Management, Peking University \\ fengzhang@ ruc.edu.cn,whitycatty@gmail.com, liuyn1999@gmail.com, albertyang33@gmail.com, \\ zhaijidong@tsinghua.edu.cn, shuhao.zhang@tu-berlin.de, hebs@comp.nus.edu.sg, linjz@pku.edu.cn, \\ duyong@ruc.edu.cn
}

\begin{abstract}
In big cities, there are plenty of parking spaces, but we often find nowhere to park. For example, New York has 1.4 million cars and 4.4 million onstreet parking spaces, but it is still not easy to find a parking place near our destination, especially during peak hours. The reason is the lack of prediction of parking behavior. If we could provide parking behavior in advance, we can ease this parking problem that affects human well-being. We observe that parking lots have periodic parking patterns, which is an important factor for parking behavior prediction. Unfortunately, existing work ignores such periodic parking patterns in parking behavior prediction, and thus incurs low accuracy. To solve this problem, we propose PewLSTM, a novel periodic weather-aware LSTM model that successfully predicts the parking behavior based on historical records, weather, environments, and weekdays. PewLSTM has been successfully integrated into a real parking space reservation system, ThsParking, which is one of the top smart parking platforms in China. Based on 452,480 real parking records in 683 days from 10 parking lots, PewLSTM yields $85.3 \%$ parking prediction accuracy, which is about $20 \%$ higher than the state-of-the-art parking behavior prediction method. The code and data can be obtained from https://github.com/NingxuanFeng/PewLSTM.
\end{abstract}

\section{Introduction}

In big cities, we often find it hard to find a parking space. However, in building cities, designers usually reserve enough parking spaces. For example, New York has at least 4.4 mil- lion on-street parking spaces ${ }^{1}$, but has only 1.4 million cars ${ }^{2}$. The reason why it is not easy to find a parking place, especially during peak hours, is the lack of prediction of parking behavior. In this paper, for a parking lot, we express parking behavior as the number of parking arrivals and departures in one hour, which can also be converted into the number of available parking spaces. Clearly, if we could provide the parking behavior in advance, we can ease such a parking problem that affects human well-being.

AI technology has transformed our everyday lives in many aspects, and now AI is shaping our parking behavior. More intelligent parking lots with AI management systems appear in our cities. Among the intelligent parking management systems, providing the parking behavior prediction is the most critical and beneficial function. First, accurate predictions can save us time and effort to find a proper parking space. For example, a motorist in New York spends an average of 107 hours per year on finding a parking space ${ }^{3}$, while such time waste could be saved when we obtain the parking behavior for the expected parking lots in advance. Second, based on the precise parking behavior prediction, the parking lots can be utilized more efficiently, and parking space reservations can also be made based on the prediction. Third, based on the parking behavior prediction, the department of transportation is better able to understand the traffic conditions and dispatch resources such as traffic police. However, parking behavior is hard to predict, since it can be affected by many factors, such as weather, weekdays, and environments.

Many intelligent parking methods and systems have been developed in recent years, such as pricing parking [Pierce and Shoup, 2013], on-street parking [Roman et al., 2018], smart

\footnotetext{
${ }^{1}$ https://www.statista.com/statistics/183505/number-of-vehiclesin-the-united-states-since-1990

${ }^{2}$ https://edc.nyc/article/new-yorkers-and-their-cars

${ }^{3}$ https://www.thedrive.com/sheetmetal/12389/new-study-showshow-much-time-and-money-we-waste-on-parking
} 
vehicle parking [Shahzad et al., 2018], and smart parking guidance [Fang et al., 2017]. However, none of these works involve parking behavior prediction. A real-time parking space availability system [Caicedo et al., 2012] has been developed, but this work only considers historical records. The state-of-the-art parking behavior prediction research [Feng et $a l ., 2019]$ used regression methods regarding parking records and weather, but we find that their method only considers parking arrivals without departures, and ignores the periodic parking patterns and thus incurs low accuracy. Even worse, they can only predict parking behavior in one hour, while we are looking forward to a long-term prediction.

Building an accurate long-term parking behavior prediction system requires overcoming three challenges. The first challenge is to find an appropriate model that can understand the complicated parking-related behaviors by using historical records and other factors such as weather and time information. The second challenge is how to involve the complex periodic parking patterns, including the influence from a specific time in the past. For example, customers would like to drive to a shopping mall every Friday night for discounts, and such patterns should be integrated. The third challenge is how to predict parking behavior for a long time in the future with the current data.

We propose PewLSTM, a periodic weather-aware LSTM model for long-term car parking behavior prediction. First, to depict the parking behavior for a parking lot, we use both the arrivals and departures, because these two indicators depict changes in parking vehicles. Second, to involve weather information, we add input gates for weather information into the LSTM block. The cell state and hidden state are decided by both previous states and weather information. Third, to utilize the periodic parking patterns from historical records, we add special gates into the LSTM block to input the hidden states from past specific time steps when certain conditions are met, such as the same time in previous weekdays.

Our proposed periodic LSTM with weather-aware gating mechanism, PewLSTM, has been integrated into a real parking system, ThsParking ${ }^{4}$ (developed by Huaching Tech ${ }^{5}$ ), which is one of the top smart parking platforms in China. Based on the prediction, the parking system can launch a segmented pricing strategy to utilize parking spaces better and gain more profit.

We evaluate PewLSTM with real parking records. We collect 452,480 parking records from 10 parking lots. These records come from different environments, including hotels, shopping malls, and streets. Along with these parking records, we have collected related weather and date information. When predicting parking behavior in the next hour, PewLSTM achieves $85.3 \%$ arrival accuracy and $84.6 \%$ departure accuracy; for the next two hours, PewLSTM achieves $76.4 \%$ arrival accuracy and $73.1 \%$ departure accuracy; and for the next three hours, its arrival accuracy is $68.6 \%$ and its departure accuracy is $68.5 \%$. On average, PewLSTM achieves $20 \%$ higher accuracy than the state-of-the-art parking prediction method [Feng et al., 2019].

\footnotetext{
${ }^{4}$ http://www.thsparking.com

${ }^{5}$ http://www.huaching.com
}

We summarize our contributions as follows.

1) We exhibit our observations, insights, and rules about the periodic patterns from different parking records, types of parking lots, and weather factors.

2) We propose PewLSTM, a periodic LSTM with weatheraware gating mechanism, which incorporates the weather and periodic parking patterns for parking behavior prediction.

3) We evaluate PewLSTM with real parking records and it achieves $85.3 \%$ accuracy, which is about $20 \%$ higher than the state-of-the-art method.

\section{Related Work}

As far as we know, this work is the first to use the LSTMbased method to predict parking behavior by using records along with weather information.

\subsection{Vehicle Parking}

Many studies have been conducted on different aspects of vehicle parking, including parking demand prediction [Alho and Silva, 2014], smart parking guidance [Yoo et al., 2008; Idris et al., 2009; Fang et al., 2017], parking space prediction [Caicedo et al., 2012; Hössinger et al., 2014; Chen, 2014; Zheng et al., 2015; Vlahogianni et al., 2016; Badii et al., 2018], and parking behavior prediction [Feng et al., 2019]. The regression-based parking behavior prediction [Feng et al., 2019] is the closest work to PewLSTM, which also considers weather conditions. They explored linear regression, ridge regression [Le Cessie and Van Houwelingen, 1992], Lasso regression [Hans, 2009], decision tree [Safavian and Landgrebe, 1991], and random forest [Liaw et al., 2002] to predict the parking arrivals in one hour, and found that random forest achieves the highest accuracy.

However, the work [Feng et al., 2019] has three limitations. First, it provides the prediction for only parking arrivals, without departures. Departures are equally important as to arrivals, and they together define the parking behavior. Second, it does not provide the prediction results for more than one hour, which limits the applicability, since users may plan to book a parking space in an uncertain future. Third, their methods fail to capture the periodic patterns from long periods of time. For example, the parking space availability may have strong correlations with previous parking patterns, such as weekday and holiday patterns. Even worse, that work used records from only one parking lot for validation, which cannot represent all parking lots. Different types of parking lots exhibit various parking behaviors, and the locations of parking lots have a high influence on parking behaviors. For example, in holidays, the parking lots near a shopping mall are busy while the parking lots in an industrial area are idle.

\subsection{Recurrent Neural Network}

Recurrent neural network (RNN) [Rumelhart et al., 1986] is a neural network that incorporates temporal sequence data as input. RNN performs recursion along the temporal sequence. Long Short-Term Memory (LSTM) [Hochreiter and Schmidhuber, 1997] is a kind of RNN and its basic unit is a cell or called LSTM block. An LSTM block is usually composed of input gates, forget gates, and output gates, which can capture the complex non-linear relations among different factors. 


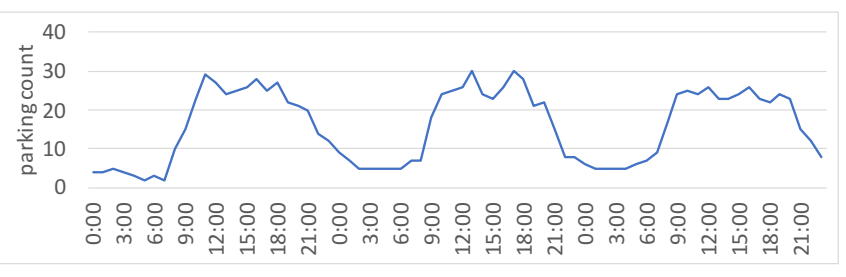

Figure 1: Periodic parking patterns for P2 at hour granularity.

LSTM has been applied to different aspects of our daily life, such as financial market prediction [Wang et al., 2019], disease progression modeling [Zhang et al., 2019], predictive phenotyping [Fu et al., 2019], text categorization [Huang et al., 2019], and sentiment analysis [Xing et al., 2019]. Particularly, LSTM conquers the limitations that the state-of-the-art parking behavior prediction method [Feng et al., 2019] failed to consider from long and short terms.

\section{Observations}

In this section, we exhibit our parking behavior observations and analysis, which are also the motivation of this work.

Data. We collect both parking and weather data. For parking data, we collect 452,480 real parking records from 10 parking lots (detailed in Section 5.1). In our study, we reduce the analysis granularity to one hour, which is the same as [Feng et al., 2019]. In this section, we use roughly the parking counts of arriving cars in the parking lot within one hour as an indicator to reflect the parking behavior. For weather information, we collect the weather data, including temperature, humidity, wind speed, and precipitation, from the national weather service.

Observation 1: Periodic Parking Patterns. Periodic parking patterns refer to that the arrivals and departures of a parking lot repeat in a cyclical and predictable manner. We find that most parking lots follow certain periodic parking patterns. For illustration, we show in Figure 1 the parking counts at hour granularity for parking lot P2 (see Table 1), which is close to a commercial street. Figure 1 shows that the parking lot follows a pattern that it is busy during the day (from 9am to $9 \mathrm{pm}$ ). The reason is that the nearby shops open at $9 \mathrm{am}$ and close at $9 \mathrm{pm}$. People go to these shops during the day and this activity lasts until the evening. Similar periodic parking patterns also exist at week and month granularities.

Observation 2: Types of Parking Lots. The types of parking lots also make the parking behaviors different from each other. For illustration, in Figure 2, we show the parking counts of two parking lots: the parking lot P4 located in an industrial park, and the parking lot P6 located near a market (see Table 1). We can see that these two parking lots show totally different behaviors. For explanation, people drive to work during working days, so P4 is busy only from Monday to Friday. In contrast, people tend to go to the market on weekends, so P6 is busy on Saturday and Sunday.

Observation 3: Influence of Different Weather Factors on Parking Behavior. The parking behavior exhibits different correlations with various weather factors. For illustration, we

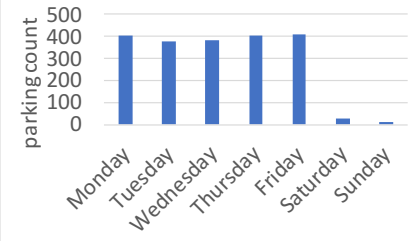

(a) P4: industrial park.

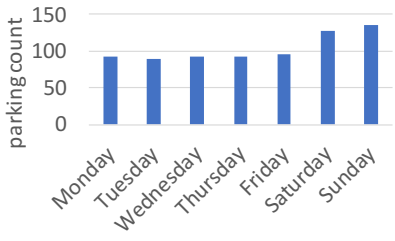

(b) P6: market.
Figure 2: Different types of parking lots.

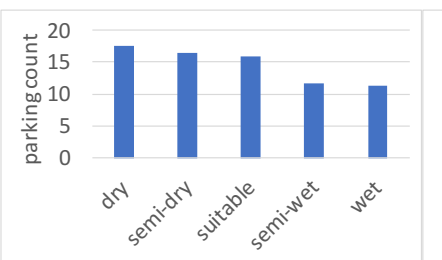

(a) Relative humidity.

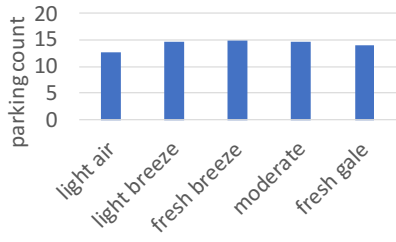

(b) Wind speed.
Figure 3: Weather influence on parking behavior for P3.

show in Figure 3 the average parking count of the parking lot P3 (see Table 1), which is close to a shopping mall. We show the relationship between the parking count and the relative humidity in Figure 3 (a), which implies that the parking behavior has a strong relationship with the relative humidity. Figure 3 (a) further implies that people are more willing to go to the mall in fine weather instead of rainy weather. We show the relationship between parking count and the wind speed in Figure 3 (b). We find that for this shopping mall, the wind speed shows less correlation with parking behavior.

\section{Parking Behavior Prediction}

\subsection{Problem Definition}

The problem we are trying to solve is how to predict the parking behavior for a given hour in the future by using the previous parking records and weather information.

Parking Behavior Definition. In this study, the parking behavior for a parking lot is defined by its arrivals and departures, as shown below. $\mathbb{N}$ represents the natural number domain.

- Arrivals. The sequence of the number of arrivals for the past $K$ time steps is $\boldsymbol{C}=\left\{c_{t-K}, \ldots, c_{t-2}, c_{t-1}\right\} \in \mathbb{N}^{K}$, where $t$ represents the current time and $c_{i}$ represents the parking number of arrivals of the past $i$-th hour.

- Departures. The sequence of the number of departures for the past $K$ time steps is $\boldsymbol{D}=$ $\left\{d_{t-K}, \ldots, d_{t-2}, d_{t-1}\right\} \in \mathbb{N}^{K}$, where $t$ represents the current time and $d_{i}$ represents the parking number of departures of the past $i$-th hour.

Significance. Different from parking space prediction, parking behavior prediction contains more information for both users and owners of parking lots. For example, a parking lot is worth waiting if the number of departures $d_{t}$ is 
large, even if the parking lot is full (parking space prediction is useless in this case since the predicted results show no vacancy). When we obtain the number of arrivals and departures for a parking lot, we can further obtain its growth of parking space usage by subtracting the amount of departures from the amount of arrivals, which is very useful for smart parking systems.

\subsection{Preliminaries}

A typical LSTM model can be formulized as the following equations, where at the time step $t, h_{t}$ represents the hidden state, $x_{t}$ represents the input vector, $c_{t}$ represents the cell sate, and $f_{t}, i_{t}$, and $o_{t}$ are the forget gate, input gate, and output gate. $W$ and $b$ are related weight matrices. Additionally, $\sigma$ indicates the sigmoid activation function, $\odot$ indicates elementwise multiplication, and $\phi$ indicates the tanh function.

$$
\begin{aligned}
\boldsymbol{f}_{t} & =\sigma\left(W_{f x} \boldsymbol{x}_{t}+W_{f h} \boldsymbol{h}_{t-1}+\boldsymbol{b}_{f}\right) \\
\boldsymbol{i}_{t} & =\sigma\left(W_{i x} \boldsymbol{x}_{t}+W_{i h} \boldsymbol{h}_{t-1}+\boldsymbol{b}_{i}\right) \\
\boldsymbol{g}_{t} & =\phi\left(W_{g x} \boldsymbol{x}_{t}+W_{g h} \boldsymbol{h}_{t-1}+\boldsymbol{b}_{g}\right) \\
\boldsymbol{o}_{t} & =\sigma\left(W_{o x} \boldsymbol{x}_{t}+W_{o h} \boldsymbol{h}_{t-1}+\boldsymbol{b}_{o}\right) \\
\boldsymbol{c}_{t} & =\boldsymbol{g}_{t} \odot \boldsymbol{i}_{t}+\boldsymbol{c}_{t-1} \odot \boldsymbol{f}_{t} \\
\boldsymbol{h}_{t} & =\phi\left(\boldsymbol{c}_{t}\right) \odot \boldsymbol{o}_{t}
\end{aligned}
$$

Challenges. We meet two challenges in applying LSTM in parking behavior prediction. The first is how to involve periodic parking patterns since typical LSTM does not have the periodic design. The second is how to design the path from sources of parking patterns and weather into the various parts within the LSTM block.

\subsection{PewLSTM Design}

In this part, we show our design of PewLSTM for parking behavior prediction and our solutions to the challenges mentioned in Section 4.2.

Model Overview. We show the model overview of PewLSTM for parking behavior prediction in Figure 4. To involve the periodic parking patterns, we add input gates for hidden states from previous LSTM functions at the same time at day, weekday, and month granularities. For example, for a time $t$, the current parking behavior may be influenced by the parking behavior at the same time from previous days (day granularity), so we add a gate for hidden states from the previous " $t-24 \cdot n$ " time step, where $n$ is a positive integer. For example, people would like to park cars at a parking lot near a restaurant at noon, and the parking behavior at noon has a strong correlation with previous behaviors at noon in the past days. The parking behavior may be influenced by the same time from last week and last month, so we add gates for inputting the time pattern influence at the same time from the past LSTM states. As for model input, each LSTM function receives both weather information and records: for weather input, we design special gates for receiving weather information, including temperature, humidity, wind speed, and precipitation; for parking record input, the record includes the arrival and departure times, date, and weekday information.

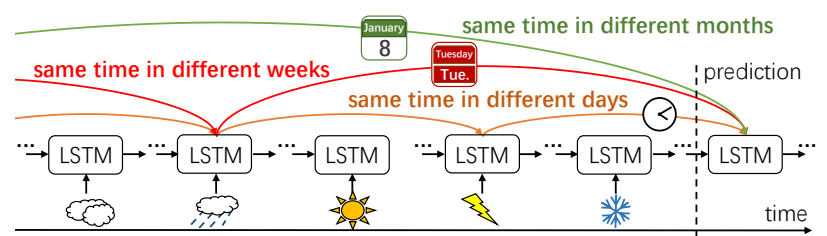

Figure 4: PewLSTM overview.

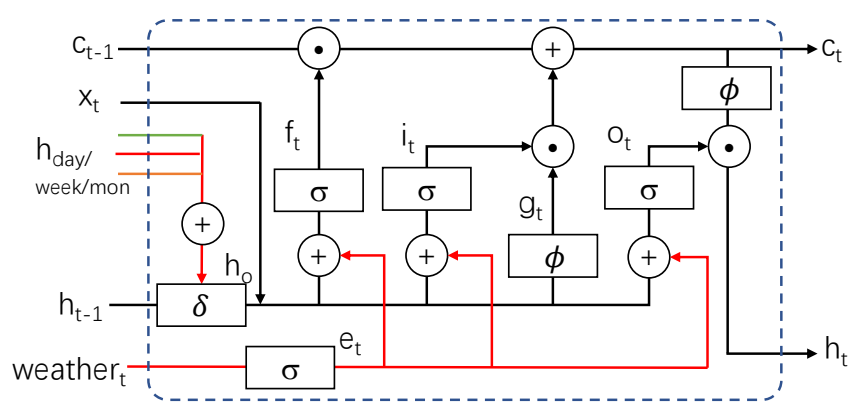

Figure 5: PewLSTM block.

PewLSTM Block. The LSTM function in PewLSTM is composed of PewLSTM blocks, as shown in Figure 5. Traditional LSTM block uses input gate $i_{t}$, output gate $o_{t}$, and forget gate $f_{t}$ to control dataflow in LSTM. We argue that for parking behavior prediction, we need to additionally involve 1) periodic pattern influence and 2) weather information. We consider these two factors as new gates involved in our PewLSTM model. We show our periodic LSTM block with weather-aware gating mechanism in Figure 5. The main difference between our PewLSTM block and traditional LSTM block lies in the gates for previous periodic records

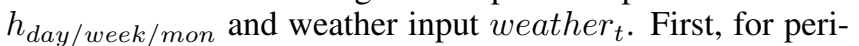
odic time influence, the hidden states at the same time from previous steps are represented as $h_{\text {day }}, h_{\text {week }}$, and $h_{m o n}$, and we design a special weight gate $\delta$ to integrate these input hidden states with $h_{t-1}$. The weight gate $\delta$ iterates continuously during training, adjusting the weight of each parameter in the model to achieve better results, as shown in Equation 2. Second, for weather influence, a weather-aware gating mechanism has been integrated into the PewLSTM block, as represented in Equation 3. We argue that the current weather information, along with the parking records and the previous parking patterns together decide the parking behaviors in the future. Based on this assumption, after a sigmoid function processing, the weather aspect vector $e_{t}$ is integrated to $f_{t}$, $i_{t}$, and $o_{t}$. Therefore, the cell state $c_{t}$ and the hidden state $h_{t}$ are influenced by the previous hidden states, cell states, the current weather information, and the periodic patterns.

$$
\begin{gathered}
\boldsymbol{h}_{o}=\delta\left(W_{d} \boldsymbol{h}_{d a y}+W_{w} \boldsymbol{h}_{w e e k}+W_{m} \boldsymbol{h}_{m o n}+W_{t-1} \boldsymbol{h}_{t-1}\right) \\
\boldsymbol{e}_{t}=\sigma\left(W_{e} \text { weather }_{t}+\boldsymbol{b}_{f}\right)
\end{gathered}
$$

Input Gates. The input gate $i_{t}$ decides the amount of data that can be accumulated to the cell state $c_{t}$ from the previous 
state $c_{t-1}$. Different from the traditional input gates, the input gate in our model integrates the input vector $x_{t}$, the weighted hidden state $h_{o}$, and the weather input $e_{t}$, as shown in Equation 4.

$$
\begin{aligned}
\boldsymbol{i}_{t} & =\sigma\left(W_{i x} \boldsymbol{x}_{t}+W_{i h} \boldsymbol{h}_{o}+W_{f e} \boldsymbol{e}_{t}+\boldsymbol{b}_{i}\right) \\
\boldsymbol{g}_{t} & =\phi\left(W_{g x} \boldsymbol{x}_{t}+W_{g h} \boldsymbol{h}_{o}+\boldsymbol{b}_{g}\right)
\end{aligned}
$$

Forget Gates. The forget gate abandons unnecessary information so that only the useful information is retained in the cell state. Similar to the input gate, along with $h_{t-1}$ and the input vector $x_{t}$, the weather information and periodic hidden states from previous time steps also affect the retention from the $c_{t-1}$ state to the $c_{t}$ state, as shown in Equation 5.

$$
\boldsymbol{f}_{t}=\sigma\left(W_{f x} \boldsymbol{x}_{t}+W_{f o} \boldsymbol{h}_{o}+W_{f e} \boldsymbol{e}_{t}+\boldsymbol{b}_{f}\right)
$$

Output Gates. The output gate is used to control the data flow from the current input to the hidden state $h_{t}$, as shown in Equation 6. Similarly, the weighted hidden state $h_{o}$, input vector $x_{t}$, and weather information together decide the output $o_{t}$, which is different from the traditional output gate.

$$
\boldsymbol{o}_{t}=\sigma\left(W_{o x} \boldsymbol{x}_{t}+W_{o h} \boldsymbol{h}_{o}+W_{f e} \boldsymbol{e}_{t}+\boldsymbol{b}_{o}\right)
$$

Cell States. Traditional cell state is decided by previous state $c_{t-1}$, forget gate $f_{t}$, input gate $i_{t}$, and $g_{t}$. We need to involve the periodic and weather information into the cell state. However, $f_{t}$ and $i_{t}$ already preserve these information. Hence, the expression of the cell state $c_{t}$ keeps the same as in Equation 1, and so does to the hidden state $h_{t}$.

Future Prediction. To predict the parking behavior in a future time step $t+k$ ( $k$ is a positive integer), we recursively predict the states in the near future, and input the predicted states into the model to predict the farther future states. Note that the periodic pattern influence also applies to the future prediction.

\subsection{Model Details}

Our PewLSTM model proposed in Section 4.3 needs further developments for different data formats.

Input Layer. The input layer receives the input data for preprocessing. In our model, the processing granularity is one hour, so parking records are converted on an hourly granularity. Along with the parking records, the input weather data, including temperature $T$, humidity $H$, precipitation $P$, and wind speed $W$, are normalized to $[0,1]$. For example, as for temperature, the corresponding sequence for the past $K$ time steps is $\boldsymbol{T}=\left\{T_{t-K}, \ldots, T_{t-2}, T_{t-1}\right\}$, where $T_{i}$ represents the temperature of the past $i$-th hour.

Deep PewLSTM Layers. Different from traditional singlelayer LSTM, we stack multiple PewLSTM layers together to form a deep neural network to capture the relations among parking records and other useful factors. Between two PewLSTM layers, the output of a PewLSTM block in one layer is the input to a PewLSTM block in the following layer.

\begin{tabular}{ccrcc}
\hline ParkingLot & Space\# & Record\# & Surrounding & Location(district/city/province) \\
\hline P1 & 23 & 22,388 & hotel & Haishu, Ningbo, Zhejiang \\
P2 & 87 & 208,472 & commercial street & Haishu, Ningbo, Zhejiang \\
P3 & 9 & 31,187 & shopping mall & Yinzhou, Ningbo, Zhejiang \\
P4 & 62 & 4,335 & industrial park & Yinzhou, Ningbo, Zhejiang \\
P5 & 46 & 28,804 & hotel & Yinzhou, Ningbo, Zhejiang \\
P6 & 16 & 32,009 & market & Yuelu, Changsha, Hunan \\
P7 & 31 & 49,707 & market & Yuelu, Changsha, Hunan \\
P8 & 27 & 46,365 & shopping mall & Yuelu, Changsha, Hunan \\
P9 & 49 & 5,808 & shopping mall & Yuhua, Changsha, Hunan \\
P10 & 65 & 23,405 & community & Yuhua, Changsha, Hunan \\
\hline
\end{tabular}

Table 1: Parking lot information.

Output Layer. Because the final output from the model is the predicted parking numbers of arrivals and departures, we add a feed-forward neural network following the deep PewLSTM layers for post-processing. Similar processes have been conducted in previous research, such as [Kong et al., 2017].

\section{Experiments}

\subsection{Experimental Setup}

Methodology. We evaluate the prediction accuracy of five methods for the future three hours. The "PewLSTM" method is our periodic LSTM with weather-aware gating mechanism based on all influencing factors. The "simple LSTM" method is an LSTM implementation based on only parking records without other factors. The "regression" method is the stateof-the-art parking behavior prediction method [Feng et al., 2019]. The "regression" method in [Feng et al., 2019] only provides results in one hour, and we extend their work for the future three hours. Additionally, we evaluate PewLSTM without periodic patterns denoted as "PewLSTM (w/o periodic)", and PewLSTM without weather information denoted as "PewLSTM (w/o weather)". Note that the algorithms involve a data preprocessing procedure to filter the noisy data and the training process has 300 epochs. The accuracy is defined in Equation 7.

$$
\text { accuracy }=1-\frac{\mid \text { count }_{\text {observed }}-\text { count }_{\text {predicted }} \mid}{\text { count }_{\text {observed }}}
$$

Datasets. In this paper, our parking datasets are composed of parking records from 10 parking lots in China, including shopping malls, hotels, communities, and so on ${ }^{6}$. We list the total number of parking spaces, parking records, surroundings, and location for each parking lot in Table 1. The dataset spans 23 months from October 16th, 2017 to August 30th, 2019 , which consists of 452,480 parking records in 683 days. For each record, we obtain the parking information including the arrival time, the departure time, date, parking space, and the price. Please note that the parking records with a duration of less than five minutes are regarded as noise data. The related weather dataset includes the hourly weather data from four districts where the 10 parking lots are built. For each hour, we collect the related weather information including temperature, wind speed, precipitation, and humidity.

\footnotetext{
${ }^{6}$ https://github.com/NingxuanFeng/PewLSTM
} 


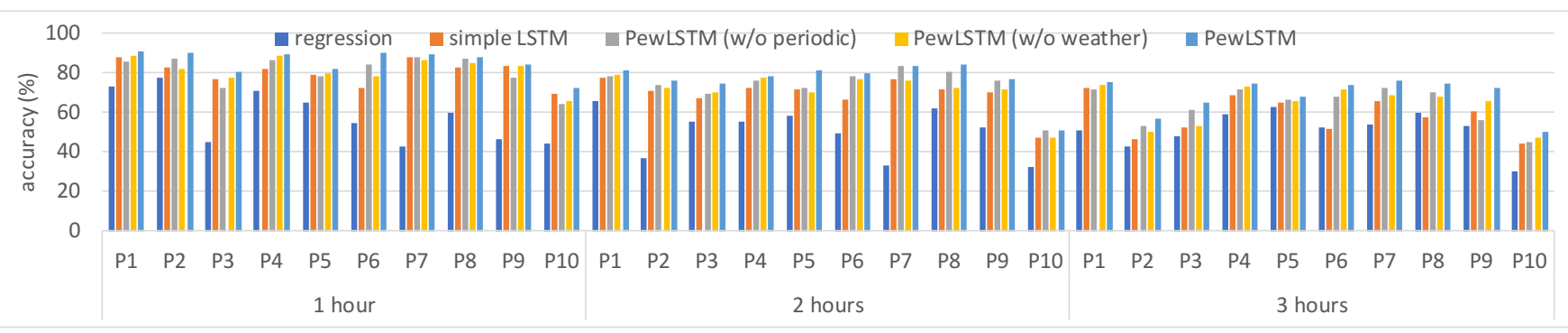

Figure 6: Arrival prediction accuracy.

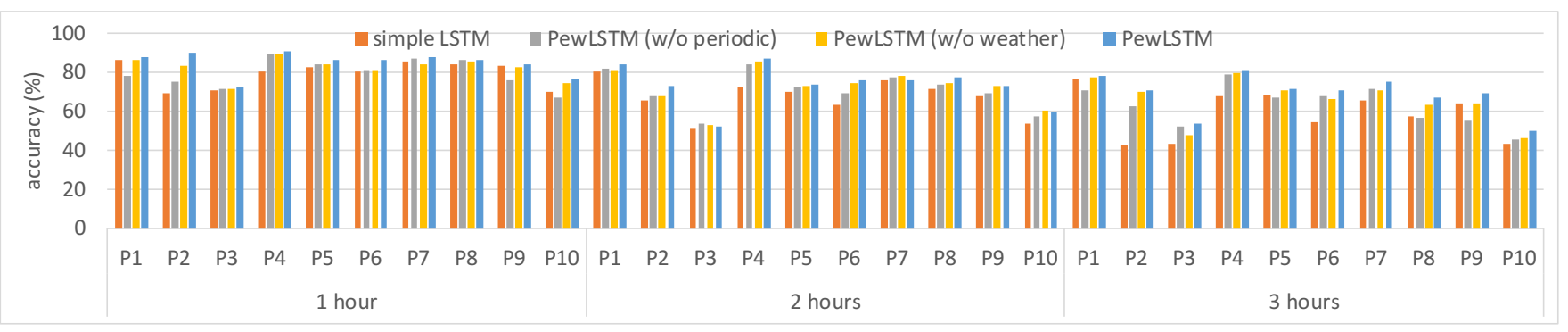

Figure 7: Departure prediction accuracy.

\subsection{Prediction Accuracy Evaluation}

We show the arrival prediction accuracy in Figure 6, and the departure prediction accuracy in Figure 7. We have the following findings.

First, our periodic LSTM with weather-aware gating mechanism achieves the highest prediction accuracy in most cases. For arrivals, PewLSTM achieves an accuracy of $85.3 \%$ in one hour, $76.4 \%$ in two hours, and $68.6 \%$ in three hours. In contrast, the regression method achieves $57.8 \%$ accuracy in one hour, $51.2 \%$ in two hours, and $50.0 \%$ in three hours. For departures, PewLSTM achieves an accuracy of $84.6 \%$ in one hour, $73.1 \%$ in two hours, and $68.5 \%$ in three hours. The "PewLSTM (w/o periodic)" and "PewLSTM(w/o weather)" achieve a moderate accuracy that is higher than the simple LSTM. This implies that periodic patterns and weather information are useful for improving prediction accuracy.

Second, with the growth of the prediction time, the prediction accuracy decreases. In our method, to predict parking behavior at a far time point, we recursively input the predicted results in a near time point into the model. We use the predicted results as training data, which inevitably introduces uncertainties and noise factors. However, our PewLSTM still maintains high accuracy in terms of long-term predictions: for every additional hour of prediction, the accuracy drops by only $8.5 \%$ for arrivals and $7.9 \%$ for departures on average.

Third, the arrival prediction accuracy is higher than the departure prediction accuracy. The reason is that departure situations are more complex than arrival situations. The departure is also affected by the purpose or event from the driver side during parking, which is hard to capture. However, even in the departure prediction, our prediction accuracy is only $1.2 \%$ lower than that in arrival prediction.

\subsection{Deployment}

Our periodic LSTM with weather-aware gating mechanism has been integrated into a real smart parking system, ThsParking, which is designed to provide users with convenient and fast parking. The system mainly provides services of online payment, smart self-parking by phones, and parking space reservation.

\section{Conclusion}

In this paper, we argue that periodic patterns shall be involved in parking behavior prediction. We have shown that parking behaviors exhibit strong periodic patterns, and such parking patterns shall be retained in classic LSTM models. To address this problem, we proposed a novel periodic LSTM with weather-aware gating mechanisms for parking behavior prediction, which considers the periodic patterns from parking records and weather data. We exhibited our model, data collection, and data processing in detail. We evaluate our proposed model with 10 parking lots in different cities, and experiments show that our model achieves $85.3 \%$ prediction accuracy, which is about $20 \%$ higher than the state-of-the-art prediction method.

\section{Acknowledgments}

This work is supported by the National Key R\&D Program of China (Grant No. 2017YFB1003103), National Natural Science Foundation of China (Grant No. 61802412, 61732014, 61972403), Beijing Natural Science Foundation (4202031, L192027), Beijing Academy of Artificial Intelligence (BAAI), Tsinghua University Initiative Scientific Research Program (20191080594), and Huaching Tech. Jidong Zhai, Jiazao Lin, and Xiaoyong Du are the corresponding authors of this paper. 


\section{References}

[Alho and Silva, 2014] André Romano Alho and João de Abreu e Silva. Freight-trip generation model: predicting urban freight weekly parking demand from retail establishment characteristics. Transportation Research Record, 2014.

[Badii et al., 2018] Claudio Badii, Paolo Nesi, and Irene Paoli. Predicting available parking slots on critical and regular services by exploiting a range of open data. IEEE Access, 2018.

[Caicedo et al., 2012] Felix Caicedo, Carola Blazquez, and Pablo Miranda. Prediction of parking space availability in real time. Expert Systems with Applications, 2012.

[Chen, 2014] Xiao Chen. Parking occupancy prediction and pattern analysis. Dept. Comput. Sci., Stanford Univ., Stanford, CA, USA, Tech. Rep. CS229-2014, 2014.

[Fang et al., 2017] Juan Fang, Aonan Ma, Haokun Fan, Min Cai, and Shuying Song. Research on smart parking guidance and parking recommendation algorithm. In 8th IEEE International Conference on Software Engineering and Service Science, 2017.

[Feng et al., 2019] Ningxuan Feng, Feng Zhang, Jiazao Lin, Jidong Zhai, and Xiaoyong Du. Statistical analysis and prediction of parking behavior. In IFIP International Conference on Network and Parallel Computing, 2019.

[Fu et al., 2019] Tianfan Fu, Trong Nghia Hoang, Cao Xiao, and Jimeng Sun. DDL: Deep dictionary learning for predictive phenotyping. In IJCAI, 2019.

[Hans, 2009] Chris Hans. Bayesian lasso regression. Biometrika, 2009.

[Hochreiter and Schmidhuber, 1997] Sepp Hochreiter and Jürgen Schmidhuber. Long short-term memory. Neural computation, 1997.

[Hössinger et al., 2014] Reinhard Hössinger, Peter Widhalm, Michael Ulm, Klaus Heimbuchner, Eike Wolf, Roland Apel, and Tina Uhlmann. Development of a realtime model of the occupancy of short-term parking zones. International Journal of Intelligent Transportation Systems Research, 2014.

[Huang et al., 2019] Ting Huang, Gehui Shen, and ZhiHong Deng. Leap-LSTM: Enhancing Long Short-Term Memory for Text Categorization. In IJCAI, 2019.

[Idris et al., 2009] MYI Idris, EM Tamil, NM Noor, Z Razak, and KW Fong. Parking guidance system utilizing wireless sensor network and ultrasonic sensor. Information Technology Journal, 2009.

[Kong et al., 2017] Weicong Kong, Zhao Yang Dong, Youwei Jia, David J Hill, Yan Xu, and Yuan Zhang. Shortterm residential load forecasting based on LSTM recurrent neural network. IEEE Transactions on Smart Grid, 2017.

[Le Cessie and Van Houwelingen, 1992] Saskia Le Cessie and Johannes C Van Houwelingen. Ridge estimators in logistic regression. Journal of the Royal Statistical Society: Series C (Applied Statistics), 1992.
[Liaw et al., 2002] Andy Liaw, Matthew Wiener, et al. Classification and regression by randomForest. R news, 2002.

[Pierce and Shoup, 2013] Gregory Pierce and Donald Shoup. Getting the prices right: an evaluation of pricing parking by demand in San Francisco. Journal of the American Planning Association, 2013.

[Roman et al., 2018] Cristian Roman, Ruizhi Liao, Peter Ball, Shumao Ou, and Martin de Heaver. Detecting onstreet parking spaces in smart cities: performance evaluation of fixed and mobile sensing systems. IEEE Transactions on Intelligent Transportation Systems, 2018.

[Rumelhart et al., 1986] David E Rumelhart, Geoffrey E Hinton, and Ronald J Williams. Learning representations by back-propagating errors. nature, 1986.

[Safavian and Landgrebe, 1991] S Rasoul Safavian and David Landgrebe. A survey of decision tree classifier methodology. IEEE transactions on systems, man, and cybernetics, 1991.

[Shahzad et al., 2018] Aamir Shahzad, Jae-young Choi, Naixue Xiong, Young-Gab Kim, and Malrey Lee. Centralized connectivity for multiwireless edge computing and cellular platform: a smart vehicle parking system. Wireless Communications and Mobile Computing, 2018.

[Vlahogianni et al., 2016] Eleni I Vlahogianni, Konstantinos Kepaptsoglou, Vassileios Tsetsos, and Matthew G Karlaftis. A real-time parking prediction system for smart cities. Journal of Intelligent Transportation Systems, 2016.

[Wang et al., 2019] Jia Wang, Tong Sun, Benyuan Liu, Yu Cao, and Hongwei Zhu. CLVSA: A convolutional LSTM based variational sequence-to-sequence model with attention for predicting trends of financial markets. In $I J$ CAI, 2019.

[Xing et al., 2019] Bowen Xing, Lejian Liao, Dandan Song, Jingang Wang, Fuzhen Zhang, Zhongyuan Wang, and Heyan Huang. Earlier attention? aspect-aware LSTM for aspect-based sentiment analysis. In IJCAI, 2019.

[Yoo et al., 2008] Seong-eun Yoo, Poh Kit Chong, Taehong Kim, Jonggu Kang, Daeyoung Kim, Changsub Shin, Kyungbok Sung, and Byungtae Jang. PGS: Parking Guidance System based on wireless sensor network. In International Symposium on Wireless Pervasive Computing, 2008.

[Zhang et al., 2019] Yuan Zhang, Xi Yang, Julie Ivy, and Min Chi. ATTAIN: attention-based time-aware LSTM networks for disease progression modeling. In IJCAI, 2019.

[Zheng et al., 2015] Yanxu Zheng, Sutharshan Rajasegarar, and Christopher Leckie. Parking availability prediction for sensor-enabled car parks in smart cities. In IEEE Tenth International Conference on Intelligent Sensors, Sensor Networks and Information Processing, 2015. 